\title{
Real-time quantitative measurement of the mode beating of an injection-seeded optical parametric oscillator
}

\author{
P. Mahnke • M. Wirth
}

Received: 5 July 2009 / Revised version: 17 November 2009 / Published online: 2 March 2010

(C) Springer-Verlag 2010

\begin{abstract}
In this paper we present the dependency of a quantitative measurement of the first-order longitudinal mode beating of an injection-seeded optical parametric oscillator (OPO) on its injection-seeding state. We show the correlation of the intensity of the first-order longitudinal mode beating and the side-mode suppression of an injectionseeded OPO. We further demonstrate that the mode-beating intensity can be used as an indicator for the spectral purity of an injection-seeded OPO.
\end{abstract}

\section{Introduction}

For many applications, especially for differential absorption lidar (DIAL), a good knowledge of the spectral properties of the transmitted radiation is mandatory [1-6]. When the bandwidth of the transmitted light is in the order of or larger than the line width of the selected absorption line, the side-mode suppression of a light source used for DIAL has a large influence on the accuracy and sensitivity of this technique. In this paper we focus on light generated by an injection-seeded optical parametric oscillator (OPO) as used in the water vapor DIAL system developed at DLR [6, 7]. The side-mode suppression (spectral purity) of an injectionseeded OPO can be monitored by measuring the transmission of the radiation through a small-bandwidth filter for

\section{P. Mahnke ( $₫)$}

Institut für Technische Physik, Deutsches Zentrum für Luftund Raumfahrt (DLR), Pfaffenwaldring 38-40, 70569 Stuttgart, Germany

e-mail: peter.mahnke@dlr.de

\section{Wirth}

Institut für Physik der Atmosphäre, Deutsches Zentrum für Luftund Raumfahrt (DLR), Oberpfaffenhofen, 82234 Wessling,

Germany the injection-seeded longitudinal mode. When the OPO is seeded on a molecular absorption line a multi-pass absorption cell can be used as the small-bandwidth filter to monitor the side-mode suppression [5]. This type of measurement has the significant drawback that it is only possible when the OPO is injection seeded on a strong absorption line, where saturation can be achieved with $100 \mathrm{~m}$ to $200 \mathrm{~m}$ of path length at a moderate vapor pressure. By measuring the first-order mode beating of the OPO we present an equivalent sensitive technique for monitoring the side-mode suppression in real time with a simple and compact setup. This technique could be employed to measure the spectral purity of lasers, too, but it should be stressed that the technique proposed here is not capable of detecting a spectral impurity due to amplified spontaneous emission.

For injection-seeded lasers the reduction/absence of mode beating is a widely used indicator for single axial (longitudinal) mode operation (see for example [8, 9]). The longitudinal mode beating measurements which are usually presented in the literature are oscilloscope images of the laser pulse, which are usually not analyzed quantitatively [8]. For direct observation of the mode beating, multi-GHz-bandwidth oscilloscopes and detectors would be needed, because OPO resonators are usually shorter than laser resonators. To be able to measure the mode-beating signals' spectral distribution quantitatively in real time with a data-acquisition card of lower bandwidth, we present a high-frequency heterodyne method to transform the spectrum of the first-order mode beating into a suitable spectral range.

In the following, we give a mathematical model describing the mode beating, which shows that the longitudinal mode beating is the sum of the spectral auto-correlations of adjacent longitudinal modes of the resonator, which is presented to our knowledge in this generalized form for the 
first time in the literature. Furthermore, we derive a relationship between the side mode suppression ratio (spectral purity) of an injection-seeded resonator and the quantitative suppression of the first-order mode beating signal intensity. Then we describe the experimental setup used to measure the mode beating and spectral purity in real time. And, finally, we present measurements which show that the quantitative measurement of the first-order mode beating is an equivalent technique compared to absorption cell measurements to measure the spectral purity of an injection-seeded optical parametric oscillator.

\section{Theory of mode beating}

\subsection{Mathematical description of mode beating of pulsed} lasers/OPOs

To understand the longitudinal mode beating of the OPO, which is measured by a fast photodiode, a closer look at the structure of the OPO's spectrum and a mathematical description of a photodiode is needed.

The photocurrent induced in the photodiode by an electromagnetic wave is proportional to the optical power irradiated onto the surface of the photodiode. For an electromagnetic wave the power flux density is described by the Poynting vector $\mathbf{S}=\mathbf{E} \times \mathbf{H}$. We assume that the detected spectrum is within a small range of the photodiode's sensitivity range and therefore we assume a constant sensitivity $R$ for the OPO radiation. In the case of our OPO the irradiated signal spectrum has a spectral width smaller than $1 \mathrm{~nm}$. The photocurrent $I_{\mathrm{ph}}$ induced in the photodiode can therefore be described as follows:

$I_{\mathrm{ph}}(t)=R\left(\iint \mathbf{S}(t, \mathbf{r}) \cdot \mathrm{d} \mathbf{A}\right) * D(t)$,

where $D(t)$ is the temporal response function of the photodiode and the spatial integration runs over the surface of the detector. The asterisk $(*)$ denotes convolution with respect to the time variable.

By Fourier transformation in the time variable and insertion of the relation between $\mathbf{S}$ and the electrical field strength $E$ for a linearly polarized vacuum wave, we get the spectral distribution of the photocurrent:

$$
\begin{aligned}
I_{\mathrm{ph}}(v)= & R D(v) \sqrt{\frac{\epsilon_{0}}{\mu_{0}}} \\
& \times \iint\left(\int E\left(v^{\prime}, \mathbf{r}\right) E\left(v^{\prime}-v, \mathbf{r}\right) \mathrm{d} v^{\prime}\right) \mathrm{d} A,
\end{aligned}
$$

where $D(v)$ is the electrical spectral response of the photodetector (for a detailed description of general photodiode response, see for example [12]) and $E(\nu, \mathbf{r})$ the electrical field of the OPO radiation on the surface of the detector. By assuming that the detector is large enough to average out the speckle patterns generated on its surface by the quasimonochromatic radiation of the OPO, it is sufficient in the following to investigate the integrand of (2) for an arbitrary $\mathbf{r}$.

Since we are not interested in absolute sensitivities, we set for the rest of this paragraph:

$I_{\mathrm{ph}}(v)=D(v)(E * E)(v)$

( $*$ denotes the convolution operator).

The spectrum emitted by a non-injection-seeded OPO is composed of a superposition of the longitudinal eigenmodes of the resonator which exceed the parametric oscillation threshold. This process is illustrated in Fig. 1. Each longitudinal eigenmode features a transversal Gauss-Hermite eigenmode structure, denoted as $\mathrm{TEM}_{m n}$, which develops in the resonator and has different resonator specific losses mainly due to the acceptance angle of the crystals. When the OPO is operated near oscillation threshold only a few longitudinal and transversal modes start to oscillate-when the OPO is operated in a saturated regime (more than five times above threshold) most longitudinal modes in the spectral acceptance range of the crystal and transversal modes in the angular acceptance range of the crystal will oscillate. The complex spectral distribution of the electrical field can be described as follows:

$E(v)=\sum_{n} E_{n}(v)$

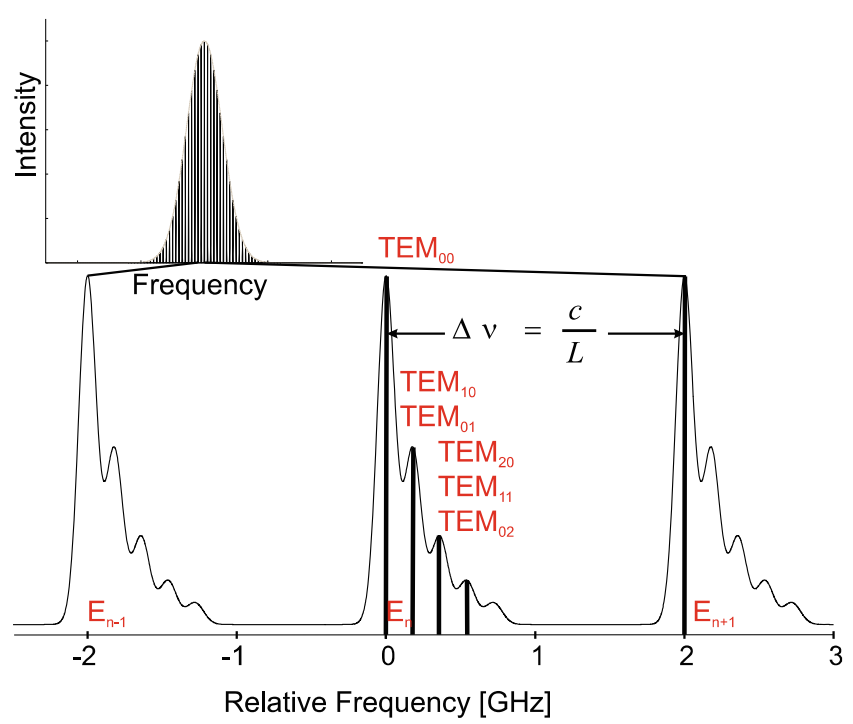

Fig. 1 Mode structure emitted by the OPO resonator. The parametric amplifier amplifies the resonator modes within the acceptance bandwidth of the crystals. The fine structure is a superposition of the different transversal modes and the line width which is defined by the ns pulse 
where $E_{n}$ is the spectral distribution of the $n$th longitudinal mode of the resonator. Being the Fourier transform of a real function, the complex spectral distribution yields the following symmetry: $E_{n}(v)=E_{n}^{*}(-v)$, where * denotes complex conjugation. Please note that a pulsed laser could have a more complicated spectral structure than a pulsed OPO due to amplified spontaneous emission, which does not occur in OPOs.

We introduce a zero frequency centered complex spectral envelope function for each longitudinal mode denoted by $A_{n}$. Using the translation $\left(\mathcal{T}_{v}\right)$ and mirroring ( ${ }^{\sim}$ ) operators defined in the appendix, we can express the complex spectral distribution as follows:

$$
\begin{aligned}
E_{n}(v, \mathbf{r}) & =A_{n}\left(v-v_{n}, \mathbf{r}\right)+A_{n}^{*}\left(-v-v_{n}, \mathbf{r}\right) \\
& =\left(\mathcal{T}_{v_{n}} A_{n}+\mathcal{T}_{-v_{n}} \tilde{A}_{n}^{*}\right)(v, \mathbf{r})
\end{aligned}
$$

where $v_{n}=n \Delta v=n c / L$ is the frequency of the $n$th longitudinal mode and $L$ is the optical resonator length.

Using the mode structure of the OPO, the spectral distribution of the photocurrent is given by

$$
\begin{aligned}
I_{\mathrm{ph}}= & D \sum_{n m} E_{n} * E_{m} \\
= & D \sum_{n m}\left[\left(\mathcal{T}_{v_{n}} A_{n}+\mathcal{T}_{-v_{n}} \tilde{A}_{n}^{*}\right)\right. \\
& \left.*\left(\mathcal{T}_{v_{m}} A_{m}+\mathcal{T}_{-v_{m}} \tilde{A}_{m}^{*}\right)\right] .
\end{aligned}
$$

By multiplying out the product in the above equation and reintroducing the variable $v$, we get

$$
\begin{aligned}
I_{\mathrm{ph}}(v)= & D(v) \sum_{n m}\left[\left(A_{n} * A_{m}\right)\left(v-v_{n}-v_{m}\right)\right. \\
& +\left(A_{n} * \tilde{A}_{m}^{*}\right)\left(v-v_{n}+v_{m}\right) \\
& +\left(\tilde{A}_{n}^{*} * A_{m}\right)\left(v+v_{n}-v_{m}\right) \\
& \left.+\left(\tilde{A}_{n}^{*} * \tilde{A}_{m}^{*}\right)\left(v+v_{n}+v_{m}\right)\right] .
\end{aligned}
$$

For the sum frequencies $\pm\left(v_{n}+v_{m}\right)$, which are in the optical region, the electrical response is $D(v)=0$; therefore, the last equation reduces to

$$
\begin{aligned}
I_{\mathrm{ph}}(v)= & D(v) \sum_{n m}\left(A_{n} * \tilde{A}_{m}^{*}\right)\left(v-v_{n}+v_{m}\right) \\
& +D(v) \sum_{n m}\left(A_{m} * \tilde{A}_{n}^{*}\right)\left(v-v_{m}+v_{n}\right) \\
= & 2 D(v) \sum_{n m}\left(A_{n} * \tilde{A}_{m}^{*}\right)\left(v-\left(v_{n}-v_{m}\right)\right) .
\end{aligned}
$$

For optical parametric oscillators with short resonators the spectral width of each individual longitudinal mode $A_{n}$ is usually smaller than the free spectral range. We therefore assume that $\left(A_{n} * \tilde{A}_{m}^{*}\right)(\nu)=0$ for frequencies larger than half of the longitudinal mode separation, i.e. $|v|>1 / 2 \Delta v{ }^{1}$ It follows that

$I_{l}(v)=2 D(v) \sum_{n}\left(A_{n} * \tilde{A}_{n-l}^{*}\right)\left(v-\left(v_{n}-v_{n-l}\right)\right)$

for frequencies $v$ which satisfy the relation $|v-l \Delta v|<$ $\frac{1}{2} \Delta v$. For $l=0$, this is the Fourier transform of the slow intensity envelope of the nanosecond impulse:

$I_{0}(v)=2 D(v) \sum_{n}\left(A_{n} * \tilde{A}_{n}^{*}\right)(v)$.

For $l \neq 0$, we can write the electrical spectrum of the $l$ th order mode beating, for positive frequencies located around $l \Delta v$, as follows:

$I_{l}(v)=2 D(v) \sum_{n}\left(A_{n} * \tilde{A}_{n-l}^{*}\right)(v-l \Delta v)$.

The frequency range around $v \approx 1 \times \Delta v$ is the first-order mode beating which is filtered, amplified and frequency transformed by the detection setup described in Sect. 3. This first-order mode beating can be interpreted as the sum of spectral auto-correlations of adjacent longitudinal modes.

For general $l$ th order, the mode-beating signal is given by

$$
\begin{aligned}
\left|I_{l}(v)\right|^{2}= & 4|D(v)|^{2} \sum_{n m}\left[\left(A_{n} * \tilde{A}_{n-l}^{*}\right)(v-l \Delta v)\right. \\
& \left.\times\left(A_{m} * \tilde{A}_{m-l}^{*}\right)^{*}(v-l \Delta v)\right]
\end{aligned}
$$

We assume that the relative phases between the different longitudinal modes of the OPO are not correlated, since it is not mode locked. Therefore, if we average the spectrum over several pulses, the products with $m \neq n$ will cancel:

$$
\left\langle\left|I_{l}(v)\right|^{2}\right\rangle \approx 4|D(v)|^{2} \sum_{n}\left|A_{n} * \tilde{A}_{n-l}^{*}\right|^{2}(v-l \Delta v) .
$$

\subsection{Comparison of mode-beating intensity and spectral purity of an (partially) injection-seeded OPO}

We define the spectral purity of an injection-seeded OPO/ laser as the ratio of the energy present in the injection-seeded

\footnotetext{
${ }^{1}$ Please note that up to this point all assumptions would hold for a pulsed laser, too. Usually, commercially available Q-switched 10-15 ns lasers have resonators with $200-300 \mathrm{MHz}$ free spectral range. Assuming that the laser emits time-bandwidth-limited Gaussian-shaped pulses with a time-bandwidth product of 0.44 , it follows that each longitudinal mode has a full width at half maximum (FWHM) spectral width of $44 \mathrm{MHz}$. This corresponds to a $20 \mathrm{~dB}$ full spectral width of $114 \mathrm{MHz}$, which is in the order of magnitude of the free spectral range of the laser. So, when small errors are acceptable, this technique could be used to diagnose lasers, too.
} 
mode $I_{\text {seeded }}$ and the total energy of the pulse $I_{\text {total }}{ }^{2}$ :

$S=\frac{I_{\text {seeded }}}{I_{\text {total }}}$

To get some qualitative insights into how the measured mode beating signal intensity compares to the spectral purity for an injection-seeded OPO, we have to make assumptions about the structure of the $A_{n}$ to simplify these relations. When the OPO is injection seeded, the broad longitudinal mode spectrum gets successively suppressed by mode competition with the injection-seeded mode [10, 13-15] dependent on many factors, such as: power of the seed laser, pump pulse length, phase mismatch of the injection-seeded mode $(\Delta k)$, spectral purity of the pump laser and resonator length (mode overlap of the injection-seeded mode with the seed laser wavelength).

The assumptions and definitions we make are as follows.

1. All longitudinal modes have a similar temporal/spectral shape $g(v)$ such that $A_{n}(v)=a_{n} g(v)$, where $a_{n}$ defines the phase and amplitude of the mode and $g(v)$ is normalized in such a way that $\int|g(v)|^{2} \mathrm{~d} v=1$. With these definitions, the total energy contained within the $n$th mode is given by

$$
P_{n} \equiv \int\left|E_{n}(v)\right|^{2} \mathrm{~d} v=2\left|a_{n}\right|^{2}
$$

2 . In the unseeded case the mode energies follow a distribution of the form $P_{n}^{u}=w_{n} P$, where $P$ is the total energy within the pulse and the $w_{n}$ are normalized weights such that $\sum_{n} w_{n}=1$.

3. In the injection-seeded case the seeded mode has the (arbitrarily chosen) index 0 and its energy is denoted by $P_{0}$. The side modes with $n \neq 0$ are assumed to have the same intensity distribution shape as in the unseeded case only with a reduced total power. Therefore, we can write

$$
P_{n}^{s}=w_{n}\left(P-P_{0}\right)=P_{n}^{u}(1-S) \text { for } n \neq 0,
$$

where $S$ is the spectral purity defined by (14).

4. The spectrum of the unseeded OPO is broad with respect to the mode separation, such that the simplifications $P_{n}^{u} \approx P_{n+1}^{u}$ and $\sum_{n} P_{n}^{u} \approx \sum_{n \neq 0} P_{n}^{u}$ hold with an accuracy of a few per cent.

Under these assumptions, the average power spectrum of the $l$ th order is given by

\footnotetext{
${ }^{2}$ We do not use the side mode suppression ratio (SMSR) defined in EN:ISO 13695, which is the ratio in $\mathrm{dB}$ of the most intensive longitudinal mode intensity and the second most intensive. For spectroscopic purposes we prefer to use the ratio of the intensity of the main (injection-seeded) longitudinal mode and the total intensity.
}

$$
\begin{aligned}
\left\langle\left|I_{l}(v)\right|^{2}\right\rangle \approx & 4|D(v)|^{2}\left|g * \tilde{g}^{*}\right|^{2}(v-l \Delta v) \\
& \times \sum_{n}\left|a_{n}\right|^{2}\left|a_{n-l}\right|^{2} .
\end{aligned}
$$

It follows that the relative suppression of mode beating by the seeding process is given by

$$
\begin{aligned}
\frac{\left\langle\left|I_{l}^{s}\right|^{2}\right\rangle}{\left\langle\left|I_{l}^{u}\right|^{2}\right\rangle} & =\frac{\sum_{n}\left|a_{n}^{s}\right|^{2}\left|a_{n+l}^{s}\right|^{2}}{\sum_{n}\left|a_{n}^{u}\right|^{2}\left|a_{n+l}^{u}\right|^{2}} \\
& =\frac{\sum_{n} P_{n}^{s} P_{n+l}^{s}}{\sum_{n} P_{n}^{u} P_{n+l}^{u}} \rightarrow \\
\frac{\left\langle\left|I_{1}^{s}\right|^{2}\right\rangle}{\left\langle\left|I_{1}^{u}\right|^{2}\right\rangle} & \approx \frac{2 P_{0} P_{1}^{u}(1-S)+\sum_{n}\left(P_{n}^{u}(1-S)\right)^{2}}{\sum_{n}\left(P_{n}^{u}\right)^{2}} \\
& =1+\left(2 \frac{w_{1}}{\sum_{n} w_{n}^{2}}-2\right) S+\left(1-2 \frac{w_{1}}{\sum_{n} w_{n}^{2}}\right) S^{2}
\end{aligned}
$$

The dimensionless factor $w_{1} / \sum_{n} w_{n}^{2}$ depends only on the intensity distribution for the unseeded case and the position of the seeded mode within this mode comb. For seeding at $\Delta k \approx 0$ and a Gaussian shape of the mode intensities, this factor is approximately $\sqrt{2}$. Is this case the relation between the mode beating signal suppression and the spectral purity is given by

$$
\begin{aligned}
\frac{\left\langle\left|I_{1}^{S}\right|^{2}\right\rangle}{\left\langle\left|I_{1}^{u}\right|^{2}\right\rangle} & =1+(2 \sqrt{2}-2) S+(1-2 \sqrt{2}) S^{2} \\
& \approx 1+0.8 S-1.8 S^{2} .
\end{aligned}
$$

\section{Experimental}

Usually, the mode beating of OPOs is not reported in the literature. This is due to the short resonators used in optical parametric oscillators, which results in mode beating in the $\mathrm{GHz}$ region. In the following, we present a method for realtime quantitative and spectral detection of the longitudinal mode beating of an OPO.

In Fig. 2 a schematic of our experimental setup is depicted. The setup consists of a walk-off-compensated injection-seeded dual-crystal single resonant ring OPO with idler dumping between the crystals, depicted on the lefthand side of Fig. 2, and a mode-beating detector, depicted on the right-hand side of the figure. The injection-seeded OPO is a slightly modified version of the OPO which we published earlier [10].

The OPO is pumped by a single longitudinal mode frequency doubled Q-switched Nd:YAG laser with a pulse length of $7.5 \mathrm{~ns}$, a repetition rate of $100 \mathrm{~Hz}, 22-\mathrm{W}$ average power at $532 \mathrm{~nm}$ and a nominal beam diameter of $5.5 \mathrm{~mm}$. The 532-nm light pumps the KTP crystals, entering the resonator, passing through mirror M1 and leaving the resonator 


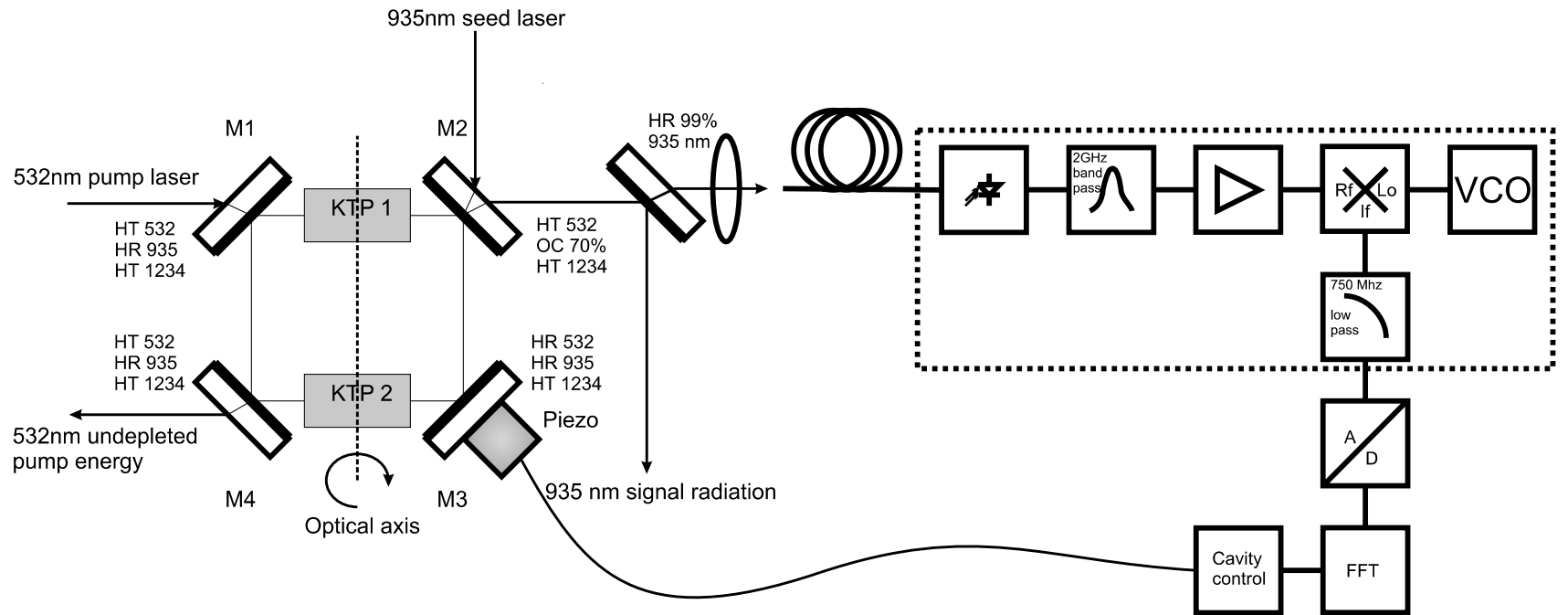

Fig. 2 Setup of the first-order mode beating experimental setup. The signal radiation of the walk-off-compensated dual-crystal injectionseeded OPO is coupled out through a $70 \%$ output coupler. A small part of the signal radiation is then measured by a fast photodetector, band-pass filtered and amplified and then transferred to the frequency

through mirror M4. Mirror M2 acts as output coupler of the 935-nm signal resonant OPO. To obtain injection seeding, the resonator length can be controlled by moving mirror M3 with a piezoelectric transducer. The 1234-nm idler light is coupled out behind each crystal to prevent a fixed phase relationship of signal, idler and pump light at the next crystal, when the OPO is injection seeded. This idler dumping also prevents back conversion in the second crystal as well as resonance effects due to the interaction between idler, signal and pump phases. The two KTP crystals were type II $(\mathrm{o} \rightarrow \mathrm{oe})$ phase matched for $935 \mathrm{~nm}$, cut under angles $\theta=70.7^{\circ}, \phi=0^{\circ}$ with an aperture of $10 \times 10 \mathrm{~mm}^{2}$, and $10-\mathrm{mm}$ length. The KTP crystals were anti-reflection coated for the pump, signal and idler radiation. To adjust the phase-matching angle $\theta$, both crystals were individually mounted on a rotation stage. The optical length of the resonator was approximately $15 \mathrm{~cm}$, which corresponds to a free spectral range of $2 \mathrm{GHz}$. For injection seeding, a fiber coupled distributed feedback (DFB) diode laser with an effective seed power of $3 \mathrm{~mW}$ was used. The DFB diode laser was stabilized onto a strong absorption line of water vapor at $935.685 \mathrm{~nm}(10687.36209 / \mathrm{cm})$. The phase mismatch $\Delta k$ of the injection-seeded mode was minimized, using a fiber-coupled spectrometer with a full width at half maximum (FWHM) resolution of $0.3 \mathrm{~nm}$, by evaluating the wavelength difference $\Delta \lambda$ between the maximum of the OPO's free running spectrum and the maximum of the spectrum of the injection-seeded mode.

Longitudinal mode beating of the OPO is expected to occur at frequencies corresponding to a multiple of the free spectral range of the OPO resonator. To observe this range between 0 and $1 \mathrm{GHz}$ by electrical mixing with the output of a voltage-controlled oscillator (VCO) (tuned to about $1.6 \mathrm{GHz}$ ). The resulting intermediate frequency is then digitized by the cavity control computer. HR: highly reflective coating, HT: highly transmitting coating, OC: output coupler

mode beating spectrally, we built a high-frequency heterodyne receiver shown on the right-hand side of Fig. 2, which transforms the spectrum of the first-order modebeating signal from $2 \mathrm{GHz}$ to $400 \mathrm{MHz}$. The radiation of the OPO signal impulse was measured using a fiber-coupled photodiode with an electrical 3-dB bandwidth in the region of $3 \mathrm{GHz}$. The first-order mode beating was then filtered by a 2-GHz band-pass filter (VBFZ-2000+, Minicircuits), which has a $2.3-\mathrm{dB}$ pass band between $1.73 \mathrm{GHz}$ and 2.27 GHz and a $20-\mathrm{dB}$ stop band starting below $1.21 \mathrm{GHz}$ and above $2.96 \mathrm{GHz}$. This filter was chosen to obtain an optimum image-frequency rejection at the downward-mixed center frequency of the first-order mode-beating signal. The filtered signal was then amplified by $20 \mathrm{~dB}$ using a low-noise amplifier for optimum sensitivity (ZEL-1724LN, Minicircuits). The amplified mode-beating signal was then mixed in a diode-ring double-balanced mixer (ZEM-4300+, Minicircuits) with a 1.6-GHz signal from a local oscillator (ZX96-2500-S+, Minicircuits). The resulting mixing product was then low pass filtered with a 780-MHz low-pass filter (VLFX-780, Minicircuits) to suppress aliasing effects in the digitizer. ${ }^{3}$ The mode-beating impulse with a center frequency at approximately $400 \mathrm{MHz}$ was then digitized using a data-acquisition system with $2-\mathrm{GHz}$ sampling rate and 1$\mathrm{GHz}$ analogue bandwidth. The rf electronic setup fits in a

\footnotetext{
${ }^{3}$ By mixing the first-order mode-beating signal with its center frequency a direct-detection receiver could be implemented. The fast digitizer could then be replaced by a boxcar integrator triggered by the laser pulse, which would result in a fully analogue real time measurement setup.
} 
shielded box of $30 \times 7 \times 3 \mathrm{~cm}^{3}$. The intensity of the mode beating was determined by calculation of the absolute square of the fast Fourier transform of the digitized signal.

Additionally, the signal radiation was analyzed by a 100-m-long multi-pass absorption cell (New Focus model 5612) not shown in Fig. 2. This absorption cell is used to determine the spectral purity of the pulsed injection seeded optical source. The power present in the injection-seeded longitudinal mode is determined indirectly by measuring the power present in the unseeded longitudinal modes. For this purpose, the absorption cell was filled with $15 \mathrm{hPa}$ of water vapor as narrow-bandwidth filter for the injection-seeded mode. The seed laser was stabilized onto a strong water vapor absorption line, where the residual transmission ratio $\tau_{\text {Cell }}$ through the cell is smaller than $10^{-5}$. The absorption line width was approximately $1.3 \mathrm{GHz}$ [11]. The spectral purity $S$ of our measurements is then given by

$S=\frac{I_{\text {seeded }}}{I_{\text {total }}}=1-\frac{I_{\text {total }}-I_{\text {seeded }}}{I_{\text {total }}} \approx 1-\tau_{\text {Cell }}$.

\section{Results}

For a better understanding of the signals, we show in the upper graph of Fig. 3 as an example a measured raw signal of the first-order mode beating measured by the dataacquisition system. The solid line which connects the measured data points is the reconstructed electrical signal. Because the first-order mode-beating signal is a bandwidthlimited signal, the signal can be reconstructed completely according to the Shannon-Nyquist theorem by convolution with a sinc kernel. In the measurement, the OPO was operated eight times above threshold and not injection seeded. The electrical signals of the first-order mode beating differ from pulse to pulse, due to the statistical nature of each individual first-order mode-beating spectrum and the arbitrary phase of the 1.6-GHz local oscillator used in the detection setup. In the lower graph of Fig. 3 the Fourier-transformed power spectrum of the first-order mode-beating signal is depicted. The spectral intensity is plotted versus the frequency, which is shifted by the frequency of the voltage-controlled oscillator (VCO in Fig. 2). To obtain a good signal to noise ratio and a fast processing time of the measured electrical signal, $\mathrm{a} \pm 16 \mathrm{~ns}$ window around the maximum of the signal is zero padded to use a fast Fourier transform to calculate the spectrum in real time. The dotted line is the spectrum of the signal shown in the upper figure. To remove the influence of the statistical nature of a single pulse mode beating spectrum, we calculated an average spectrum of 20 consecutive unseeded OPO pulses plotted as the solid line in the lower graph. From the theory, one would expect perfectly symmetric spectra, because the first-order mode beating is
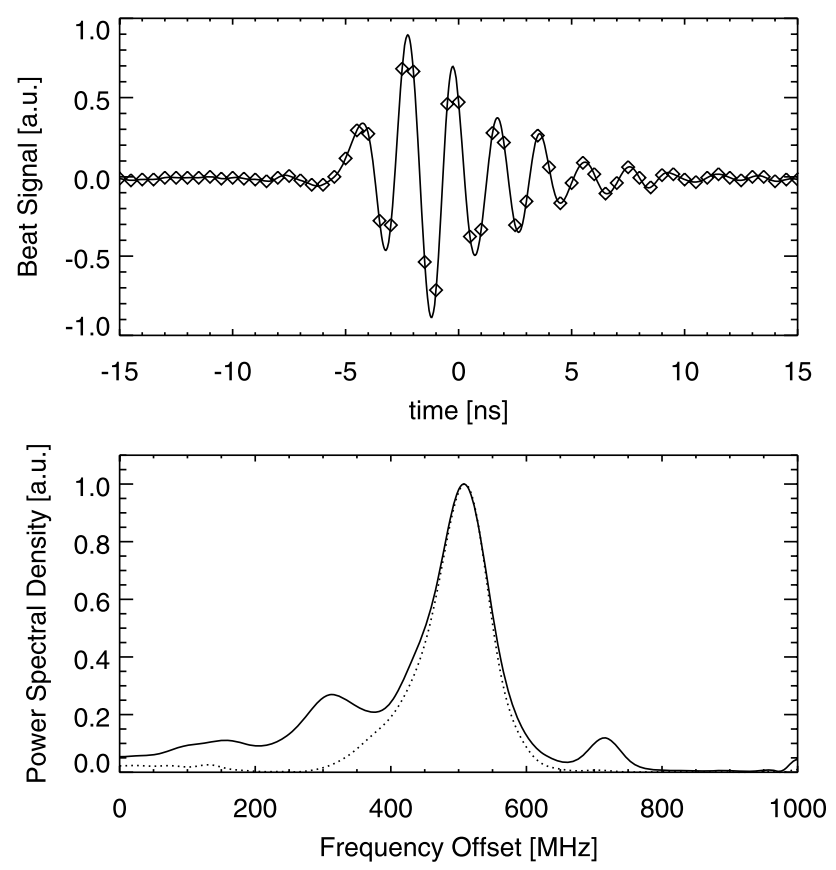

Fig. 3 In the upper figure the sampled signal generated by our receiver circuit for an arbitrary unseeded OPO pulse is shown. The OPO was operated eight times above threshold. The dotted line in the lower figure shows the Fourier-transformed spectrum of this pulse. The solid line shows the averaged heterodyne shifted first-order mode-beating spectrum of 20 unseeded OPO pulses

the sum of the temporal auto-correlations of adjacent longitudinal modes of the resonator. The distortion observed in the measured spectra is due to the frequency response of the components used (photodiode, filters, amplifier and mixer). Because this frequency response is a linear effect in the frequency domain, it does not affect the quantitative measurement of the mode beating. The distortion could be compensated by multiplying the measured frequency response of the setup with the measured spectrum. For distortion-free observation of the first-order mode beating, a fast oscilloscope with at least 3-GHz bandwidth could be used, but this would not enable real-time $100-\mathrm{Hz}$ quantitative measurements of the first-order mode beating to measure the spectral purity of an (partially) injection-seeded optical parametric oscillator. On the other hand, a better observation of longitudinal-transversal mode-beating effects of pulsed (not injection-seeded) optical parametric oscillators could be studied in more detail, as described in the following paragraph.

Taking a closer look at the averaged spectrum, we can observe the frequency shift of the higher-order transversal modes of the OPO resonator. The frequency difference of the higher-order transversal modes which is observed is approximately $160 \mathrm{MHz}$. The frequency difference of the higher-order transversal modes could stem from thermal lenses in the OPO crystals (or other nonlinear phase 


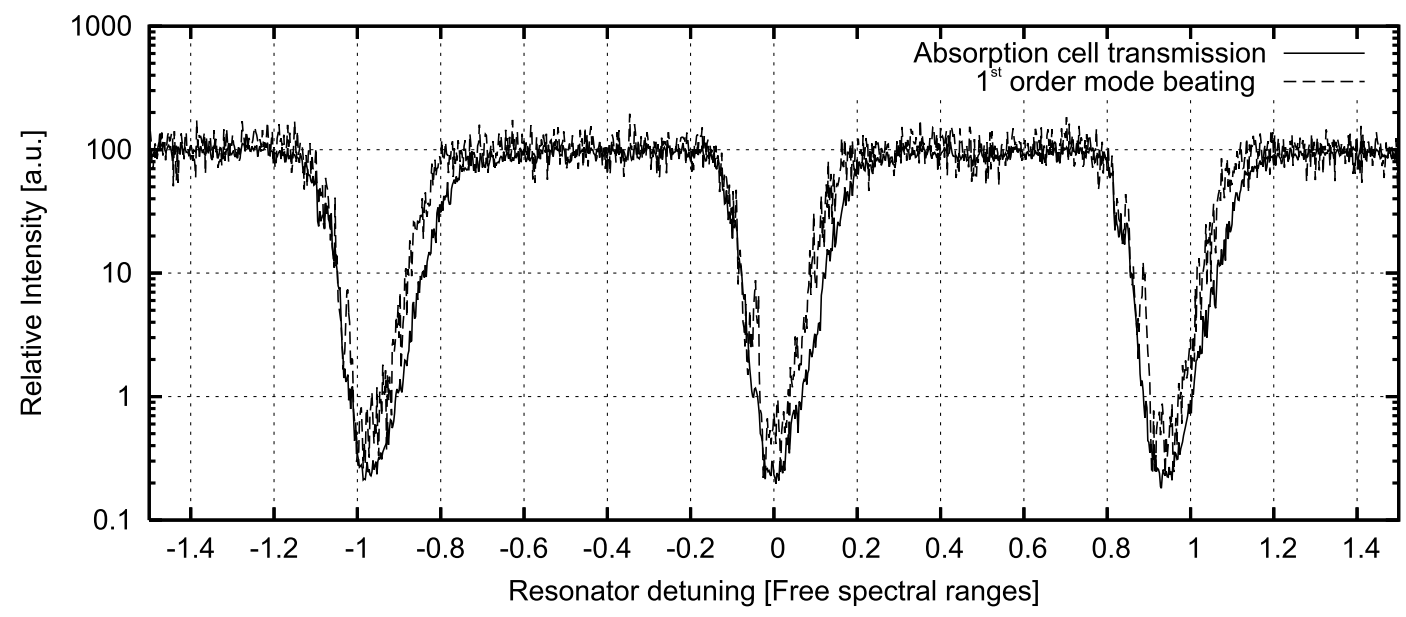

Fig. 4 Plot of the resonator length dependency of the absorption cell transmission and the first-order side-mode intensity. During the resonator length scan the OPO was operated 3.5 times above threshold

distortions) and can be derived from formulas of classical resonator theory [16]: $\delta v_{\text {transversal }}=\delta v * \arccos (1-L / R)$. In the presented case the frequency shift corresponds to a phase distortion of a resonator internal lens with 2-m focal length $(R=4 \mathrm{~m})$. The distortion which can be observed in the measured spectrum is due to the transient behavior of the band-pass and low-pass filters present in the detection scheme. For a fast analysis and comparison with the absorption cell transmission (spectral purity), we use in the following measurements only the maximum peak of this spectrum for different injection-seeding states of the OPO.

In Fig. 4 the relative transmission of an OPO signal pulse through the multi-pass cell and the relative sidemode spectral intensity are plotted for a scan of the resonator length. The OPO was operated approximately 3.5 times above threshold during the measurement. Scanning the resonator length of the OPO shifts the resonator mode comb over several free spectral ranges. When a resonator mode approaches the wavelength of the injection seeder the injection-seeding process suppresses by mode competition the free running OPO spectrum which is transmitted through the absorption cell and at the same time the firstorder mode beating is suppressed. A good correlation between the first-order mode-beating signal and the absorption cell signal can be observed. To see the proportionality between the signals, we need to calculate $1+0.8 S-S^{2}$ from the absorption cell transmission. This comparison is presented in Fig. 5. We observe an excellent correlation between the two curves. The only drawback is that the firstorder mode beating signal exhibits more noise, due to the statistical nature of the phases between two adjacent longitudinal modes, which has no influence on the absorption cell transmission signal.

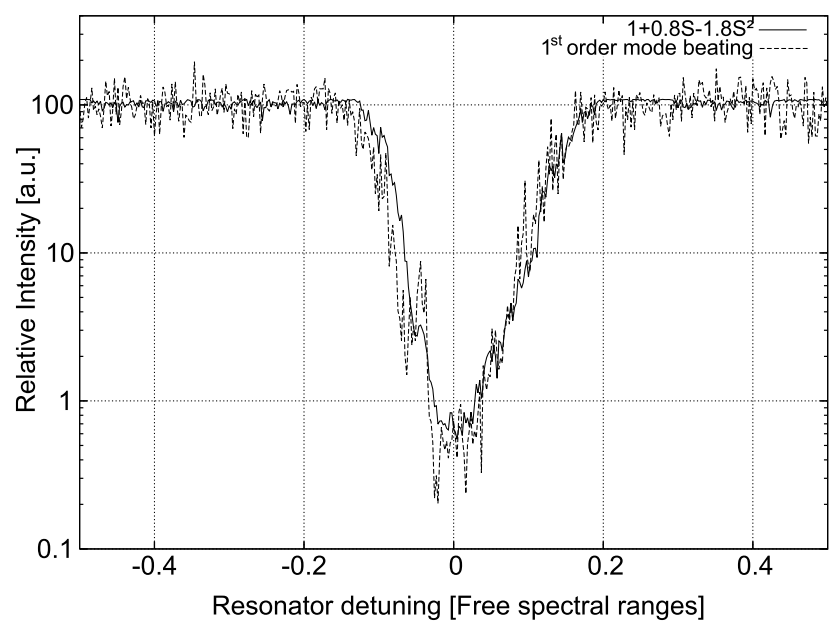

Fig. 5 Plot of the resonator length dependency of $1+0.8 S-S^{2}$ calculated from the absorption cell transmission and the first-order side-mode intensity. The OPO was operated 3.5 times above threshold during this scan

\section{Summary}

We demonstrated a technique to quantitatively measure the first-order mode beating of an OPO by a simple, inexpensive and compact high-frequency electronic circuit. We believe that this is a powerful diagnostic tool to monitor the spectral properties of an injection-seeded OPO, where no other spectral monitoring can be applied. Additionally, we think that the detection of the first-order mode beating can also be applied to injection-seeded lasers as well, but further research will be necessary to evaluate the case of spectrally overlapping adjacent longitudinal mode spectra. 


\section{Appendix: translation and convolution}

To simplify the notation of the spectra, we define the following operators:

$\mathcal{T}_{a} f(x) \equiv f(x-a) \quad$ shift operator,

$\tilde{f}(x) \equiv f(-x) \quad$ mirror operator.

The convolution is defined by

$(f * g)(x) \equiv \int_{-\infty}^{\infty} f(y) \cdot g(x-y) \mathrm{d} y$.

For the convolution and the shift operator, the following relations apply:

$\left(\mathcal{T}_{a} f\right) * g=\mathcal{T}_{a}(f * g)=g *\left(\mathcal{T}_{a} f\right)$.

For the shift operator, the following additional relations are valid:

$\widetilde{\mathcal{T}_{a} f}=\mathcal{T}_{-a} \tilde{f}$,

$\mathcal{T}_{a} \mathcal{T}_{b} f=\mathcal{T}_{a+b} f$
2. A. Ansmann, J. Bösenberg, Appl. Opt. 26, 3026 (1987)

3. J. Bösenber, Appl. Opt. 37, 3845 (1998)

4. E.V. Browell, S. Ismail, W.B. Grant, Appl. Phys. B 67, 399 (1998)

5. G.K. Schwemmer, M. Dombrowski, C.L. Korb, J. Milrod, H. Walden, R.H. Kagann, Rev. Sci. Instrum. 58, 2226 (1987)

6. G. Poberaj, A. Fix, A. Assion, M. Wirth, C. Kiemle, G. Ehret, Appl. Phys. B 75, 165 (2002)

7. M. Wirth, A. Fix, P. Mahnke, H. Schwarzer, F. Schrandt, G. Ehret, Appl. Phys. B 96, 201 (2009)

8. L.A. Rahn, Appl. Opt. 24, 940 (1985)

9. R.L. Schmitt, L.A. Rahn, Appl. Opt. 25, 629 (1985)

10. P. Mahnke, H.H. Klingenberg, A. Fix, M. Wirth, Appl. Phys. B 89, 1 (2007)

11. L.S. Rothman, D. Jacquemart, A. Barbe, D.C. Benner, M. Birk, L.R. Brown, M.R. Carleer, C. Chackerian, K. Chance, L.H. Coudert, V. Dana, V.M. Devi, J.M. Flaud, R.R. Gamache, A. Goldman, J.M. Hartmann, K.W. Jucks, A.G. Maki, J.Y. Mandin, S.T. Massie, J. Orphal, A. Perrin, C.P. Rinsland, M.A.H. Smith, M.J. Tennyson, R.N. Tolchenov, R.A. Toth, J. Vander Auwera, P. Varanasi, G. Wagner, J. Quant. Spectrosc. Radiat. Transfer 96, 139 (2005)

12. T.N. Swe, K.S. Yeo, An accurate photodiode model for DC and high frequency SPICE circuit simulation, in Nanotech 2001, vol. 1 , p. 362

13. A. Fix, R. Wallenstein, J. Opt. Soc. Am., B, Opt. Phys. 13, 2484 (1996)

14. G. Anstett, R. Wallenstein, Appl. Phys. B 79, 827 (2004)

15. E.S. Cassedy, M. Jain, IEEE J. Quantum Electron. QE-15, 1290 (1979)

16. H. Kogeknik, T. Li, Appl. Opt. 5, 1550 (1966)

\section{References}

1. A. Ansmann, Appl. Opt. 24, 3476 (1985) 